\section{Acute osteomyelitis of the acetabulum induced by Staphylococcus capitis in a young athlete}

\author{
Seiji Fukuda, Keisuke Wada, Kenji \\ Yasuda, Junji Iwasa,' Seiji Yamaguchi \\ Department of Pediatrics, and \\ 'Department of Orthopedic Surgery, \\ Shimane University School of Medicine, \\ Shimane, Japan
}

\section{Abstract}

Acute hematogenous osteomyelitis (AHOM) of the acetabulum is a rare condition in children and usually caused by Staphylococcus aureus. We present an 11-year-old soccer athlete who suffered from acute osteomyelitis involving the acetabulum caused by $S$. capitis, a normal flora of the human skin but never reported in this condition. The disease was associated with repetitive skin injuries of the knee and potential osseous microtrauma of the hip joint by frequent rigorous exercise. This unusual case suggests that osseous microtrauma of the acetabulum, in addition to repetitive skin injuries, allowed normal skin flora to colonize to the ipsilateral acetabulum, which served as a favorable niche and subsequently led to AHOM.

\section{Introduction}

Acute hematogenous osteomyelitis (AHOM) in children usually occurs in tubular bones. In contrast, $\mathrm{AHOM}$ of the pelvis represents only $7-9 \%$ of AHOM cases., ${ }^{1,2}$ The most common focus of pelvic ostemomyelitis is the ilium (38-40\%), followed by the ischium (19-28\%), pubis (14-15\%), and acetabulum (7-12\%).,3 The frequency of involvement of the acetabulum is estimated at $0.5-1.1 \%$ of the total childhood AHOM cases. Pre-existing trauma is an uncommon event in pelvic osteomyelitis compared to AHOM of the tubular bones. ${ }^{3}$ We present the case of a young male athlete who suffered from acute pelvic osteomyelitis caused by $S$. capitis, a normal flora of the human skin that has never been reported in this condition. The disease was associated with repetitive skin injuries of the knee and potential osseous microtrauma of the acetabulum by rigorous exercise, which we believe are responsible for colonization of normal flora to the acetabulum.

\section{Case Report}

An 11-year-old boy without any remarkable history of illness visited our emergency department complaining of high fever and mild pain in his left thigh. His body temperature was $39-40^{\circ} \mathrm{C}$ for two days prior to hospitalization. His throat was slightly infected, but the chest X-ray did not indicate signs of pneumonia. Mild pain and tenderness on the back of the left femur, with minimum limitation of motion of the hip joint were found, but the femoral X-ray finding was unremarkable. Blood laboratory tests taken the following day indicated normal white blood cell (WBC) count $(8,960 / \mu \mathrm{L})$ and mild elevation of $\mathrm{C}$ reactive protein (CRP) $(3.27 \mathrm{mg} / \mathrm{dL})$, but there was a marked increase of the erythrocyte sedimentation rate (ESR) (67/hr). Procalcitonin, serum amyloid A, MMP-3, renal function, and urinary analysis were all normal. Influenza virus and adenovirus tests of the throat swab were negative. Remittent fever persisted for another seven days, but gradually returned to normal by day 8 without any medication.

However, his femoral pain became unbearable on day 6 and localized tenderness of the thigh and hip joint became evident. After asking questions about his exercise history, we discovered that he was a very active soccer player and constantly kicked the ball using his left leg as his pivoting leg while playing soccer. In addition, he had injured his left knee during a game about two weeks before hospitalization and reinjured this lesion in a similar manner a week later. Although the wound had been disinfected, excessive discharge from the skin had been noticed. The wound on the left knee was covered up by skin at the time of hospitalization. While the pelvic $\mathrm{X}$ rays showed no remarkable findings, the T2MRI image of the pelvis taken on day 8 demonstrated an intensified bright signal on the left acetabulum, ilium, and ischium (Figure 1).

${ }^{67} \mathrm{Ga}$ scintigraphy scanned on day 11 also showed increased isotope uptake by the same lesion (Figure 2). S. capitis was identified in the blood culture, but was sensitive to cephalosporin and penicillin. These findings led to the diagnosis of acute osteomyelitis of the acetabulum, ilium, and ischium.

Although local tenderness and fever resolved and CRP spontaneously returned to negative, ESR remained elevated. Because delay in treatment may result in long-term morbidity, ${ }^{3}$ intravenous cefazolin sodium was administered for 10 days until day 21. ESR decreased to the normal range by day 21 without recurrence of a febrile episode. The abnormal MRI signal around the acetabulum persisted at the time of discharge, but no
Correspondence: Seiji Fukuda, Department of Pediatrics, Shimane University School of Medicine, 89-1 Enya Cho, Izumo, 693-8501, Japan. E-mail: sfukuda@med.shimane-u.ac.jp

Key words: acute osteomyelitis, acetabulum, osseous microtrauma, pre-existing trauma, Staphylococcus capitis.

Contributions: SF participated in the patient's care, interpreted the laboratory data, and wrote the manuscript; KW, KY, JI participated in the patient's care and analyzed the laboratory data; SY organized and directed the study. All authors have read and approved the final version of the manuscript.

Acknowledgements: we thank Mr. John Telloyan for technical assistance in editing this manuscript.

Conflict of interest: the authors report no conflicts of interest. No funding source is involved and IRB approval is not applicable.

Received for publication: 24 December 2009. Accepted for publication: 3 February 2010.

This work is licensed under a Creative Commons Attribution 3.0 License (by-nc 3.0).

(C) Copyright S. Fukuda et al., 2010

Licensee PAGEPress, Italy

Pediatric Reports 2010; 2:e2

doi:10.4081/pr.2010.e2

longer existed at four months. Thigh pain and fever never recurred.

\section{Discussion}

The diagnosis of acute pelvic osteomyelitis generally is established by the presence of two or more of the following signs: local signs of inflammation, positive culture results from either blood or needle aspirate of bone, and typical radiographic changes or a positive isotope scan. ${ }^{3}$ Our case was diagnosed based on these findings in addition to remittent fever, elevated ESR, and an intensified T2-MRI signal, generally indicative of bone edema. ${ }^{4}$ Preexisting trauma has been reported in 17-26\% of cases with pelvic acute osteomyelitis, ${ }^{2,3}$ in contrast to the $30-40 \%$ incidence rate of preceding trauma in classical childhood acute osteomyelitis of tubular bones, which could be attributed to the trauma-resistant nature of the pelvic bones in children. ${ }^{3}$ Our patient was a very active soccer player who exercised rigorously, but injured the same lesion on his left knee two consecutive weeks before manifesting symptomatic AHOM. The skin lesion resulting from repetitive knee injury would 

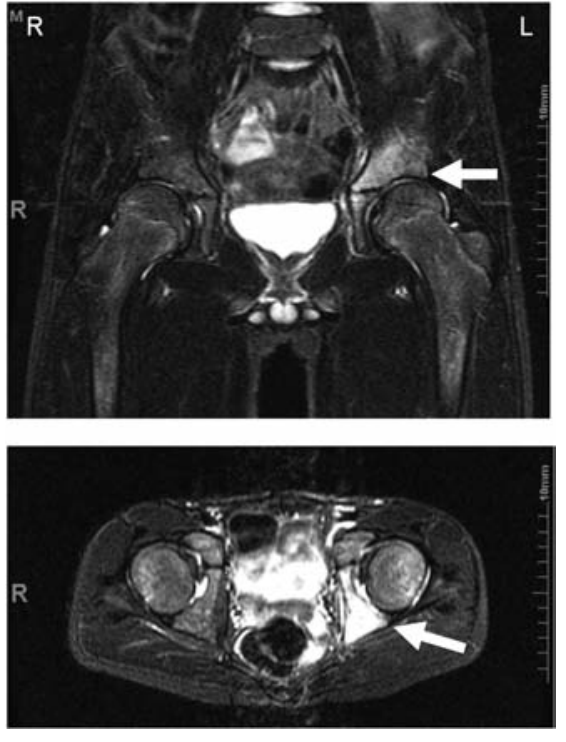

Figure 1. Magnetic resonance imaging (T2) of the pelvis taken on day 8 of hospitalization. High-intensity signal was detected on the left acetabulum, ilium, and ischium. The top and bottom panels represent the frontal and horizontal views, respectively.

have served as a port of entry for the pathogen on the skin. The most common pathogen for pelvic osteomyelitis is $S$. aureus. $^{1-3,5}$ His blood culture indicated the existence of $S$. capitis, a normal flora of the human skin that can cause serious infectious disease, such as endocarditis, pneumonia, or urinary tract infection, ${ }^{6}$ but had never been reported in pelvic AHOM.

While pelvic osteomyelitis in a young athlete has not been reported very often, Seve $e t$ al. suggested that osseous microtrauma in athletes can act as a risk factor for seeding of pathogens during transient bacteremia and subsequent osteomyelitis. ${ }^{7}$ However, we know of no clinical report that substantiates this hypothesis, and our patient appears to be the first reported case of AHOM of the acetabulum that supports this proposal. He is a very active soccer player who has a left pivoting leg. This suggests that his left acetabulum was repeatedly subjected to strong physical pressure against the rotating force created by his body weight whenever he kicked the ball with his right leg. This would increase the chance of microtrauma of the acetabulum by

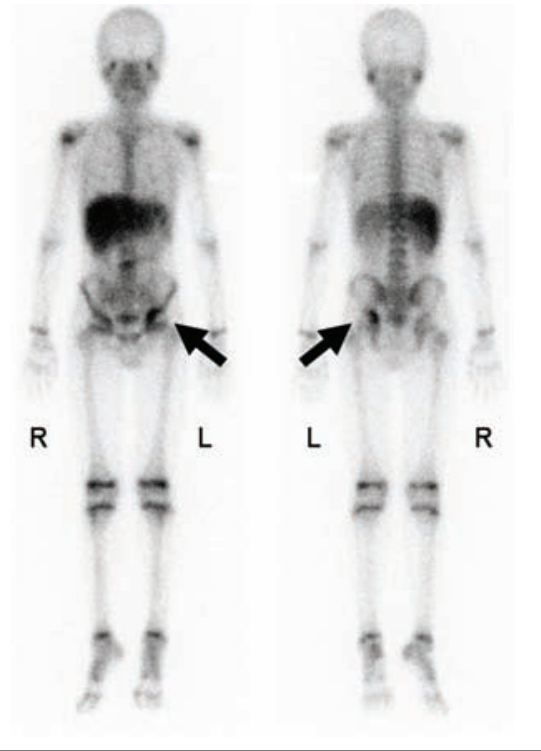

Figure 2. ${ }^{67} \mathrm{Ga}$ scintigraphy scanned on day 11. Increased isotope uptake by the left hip joint was observed. Arrows indicate the abnormal signal. $R$ and $L$ represent right and left, respectively.

the femoral head. It is highly unlikely that the normal skin flora induces AHOM in healthy individuals. Nevertheless, the osseous microtrauma would have allowed flora that are normally non-pathogenic to colonize in the acetabulum, which served as a favorable niche and subsequently induced AHOM. However, our patient's fever resolved spontaneously before treatment with antibiotics. Since $S$. capitis is normally non-virulent in the non-immune compromised host like our case, it is highly likely that the local bacterial growth was limited and captured by the host immune system relatively quickly.

The pain of the pelvic osteomyelitis is often referred to the ipsilateral hip or thigh, leading to misdiagnosis in about two out of three cases, or a delay in the correct diagnosis., The diagnosis of osteomyelitis was delayed in our patient because the history of rigorous exercise and recent injury was not recognized until the left femoral pain became evident. After a patient's wounds are healed, skin injuries may not be fully appreciated by physicians unless they ask the correct, relevant questions.

\section{Conclusions}

We experienced the case of a boy with acute pelvic osteomyelitis caused by $S$. capitis, a normal flora of the human skin but never reported in this condition. The disease was associated with repetitive skin injuries and potential osseous microtrauma. We suspect that the combination of occult bacteremia resulting from repetitive skin injury of the knee and the pre-existing osseous microtrauma of the acetabulum caused by physical force during rigorous exercise allowed normal flora to colonize to the acetabulum, which in turn induced AHOM of the pelvis. As presented here, the diagnosis of osteomyelitis of the pelvis is often delayed or misdiagnosed in patients suffering from hyperpyrexia with an unidentified focus. In addition, physicians should be aware of AHOM involving the acetabulum as a potential diagnosis for prolonged fever of unknown origin, especially in young athletes experiencing rigorous exercise.

\section{References}

1. Saavedra-Lozano J, Mejias A, Ahmad N, et al. Changing trends in acute osteomyelitis in children: impact of methicillin-resistant staphylococcus aureus infections. J Pediatr Orthop 2008; 28:569-75.

2. Weber-Chrysochoou C, Corti N, Goetschel P, et al. Pelvic osteomyelitis: a diagnostic challenge in children. J Pediatr Surg 2007; 42:553-7.

3. Zvulunov A, Gal N, Segev Z. Acute hematogenous osteomyelitis of the pelvis in childhood: Diagnostic clues and pitfalls. Pediatr Emerg Care 2003;19:29-31.

4. McPhee E, Eskander JP, Mahan ST, et al. Imaging in pelvic osteomyelitis: support for early magnetic resonance imaging. J Pediatr Orthop 2007;27:903-9.

5. Klein DJ, Leach KA. Pediatric pelvic osteomyelitis. Clin Pediatr 2007;46:787-90.

6. Mainardi JL, Lortholary 0, Buu-Hoi A, et al. Native valve endocarditis caused by Staphylococcus capitis. Eur J Clin Microbiol Infect Dis 1993;12:789-91.

7. Seve P, Boibieux A, Pariset C, et al. Pubic osteomyelitis in athletes. Rev Med Interne 2001;22:576-81. 